\title{
Mathematical Modeling of Unsteady Gas Transmission System Operating Conditions under Insufficient Loading
}

\author{
Vasyl Zapukhliak ${ }^{1}$, Lyubomyr Poberezhny ${ }^{1}$, Pavlo Maruschak ${ }^{2}{ }^{\circledR}$, Volodymyr Grudz Jr. ${ }^{1}$, \\ Roman Stasiuk $^{1}$, Janette Brezinová ${ }^{3, *}$ and Anna Guzanová ${ }^{3}$ \\ 1 Department of Construction and Renovation of Oil and Gas Pipelines and Oil and Gas Storage Facilities, \\ Ivano-Frankivsk National Technical University of Oil and Gas, Ivano-Frankivsk, Karpatska str. 15, \\ 76019 Ivano-Frankivsk, Ukraine; psmal@protonmail.com (V.Z.); rmax2019@bigmir.net (L.P.); \\ ndl-pzism@meta.ua (V.G.J.); ndr-prism@meta.ua (R.S.) \\ 2 Department of Industrial Automation, Ternopil National Ivan Pul'uj Technical University, Ruska str. 56, \\ 46001 Ternopil, Ukraine; maruschak.tu.edu@gmail.com \\ 3 Department of Engineering Technologies and Materials, Faculty of Mechanical Engineering, Technical \\ University of Košice, Mäsiarska 74, 04001 Košice, Slovakia; anna.guzanova@tuke.sk \\ * Correspondence: janette.brezinova@tuke.sk; Tel.: +421-55-602-3542
}

Received: 12 March 2019; Accepted: 3 April 2019; Published: 6 April 2019

\begin{abstract}
Under insufficient loading of a main gas transmission system, high-amplitude fluctuations of pressure may occur in it. A mathematical model is proposed to estimate the amplitude of pressure fluctuations in a gas pipeline along its length. It has been revealed that the shutdown of compressor stations along the gas pipeline route has a significant impact on the parameters of the unsteady transient operating conditions. The possibility of minimizing oscillation processes by disconnecting compressor stations is substantiated for the "Soyuz" main gas pipeline.
\end{abstract}

Keywords: main gas pipeline; pressure fluctuations; unsteady process

\section{Introduction}

At its core, the design of any gas transmission system is based on the carrying capacity (throughput) of the gas pipeline defined as the maximum amount of gas that can be pumped per unit of time. This value is decisive when choosing the diameter and number of compressor stations, working pressures at their inlet/outlet limited by the pipe strength, and so on. The lower limit of these parameters is chosen from the normal operating conditions of gas pumping units [1-3]. Maximum and minimum pressures have an impact on the carrying capacity: the smaller the difference between them, the lower the performance, and vice versa.

Under conditions of insufficient loading of the gas transmission system, a significant decrease in performance compared with the throughput leads to a broader range of maximum and minimum pressures. Boundary variants of admissible regimes include the maximum one, which is characterized by the maximum pressure at the beginning of the linear section, and the minimum one, which is characterized by the minimum pressure at the end of the linear section. All other admissible regimes are in the range between the specified boundaries [3-5].

Papers which describe mathematical models of pressure fluctuations in the gas transmission system due to changes in performance characteristics under insufficient loading are known. The realization of such models under real operating conditions of gas pipelines allowed establishing the amplitude and frequency characteristics of the unsteady process [6,7]. It was found that in the low-frequency region, the pressure fluctuation amplitude can exceed 1.0 MPa. As a result, the absolute 
value of pressure goes beyond the admissible range. In addition, it should be noted that propagation rates of disturbances in the gas pipeline at high and low pressures differ significantly, which affects frequency characteristics of the unsteady process. Summarizing the foregoing, it should be emphasized that, despite the economy yielded by the transmission of gas at high pressures, it is advisable to leave a certain margin of possible amplitudes of pressure fluctuation in order to prevent the attainment of the limiting absolute values of pressure [8]. This paper focuses on determining permissible fluctuations of pressure in the insufficiently loaded gas transmission system based on mathematical models.

\section{Research Technique}

It should be noted that under conditions of insufficient loading of the gas transmission system, the only optimality criteria of operating conditions can be the minimum energy consumption in the process of gas transportation and the maximum reliability of the gas pipeline. Based on the first criterion, we define the principle of optimizing the above admissible regimes [9-11]. Consider a gas pipeline with an internal diameter $d$, which contains two compressor stations, with the pressure at the inlet and outlet $P_{H i}, P_{B i}$, respectively, and two linear sections with lengths $L_{i}$. The throughput of each section as a function of parameters of the operating conditions is the main indicator of performance, which characterizes the intended use of the pipe, and is determined from the basic equation of the gas pipeline [12-14]:

$$
\begin{gathered}
Q=0.326 \cdot 10^{-6} \cdot d_{i}^{2.5} \cdot \sqrt{\frac{P_{H}^{2}-P_{K}^{2}}{\lambda_{i} \Delta z T_{c p} L_{i}}} \\
\lambda_{i}=0.067\left(\frac{158}{R e_{i}}+\frac{2 K_{l}}{d_{i}}\right)^{0.2} \\
\operatorname{Re}_{i}=1.81 \cdot 10^{3} \frac{Q_{\Delta}}{d_{i} \eta}
\end{gathered}
$$

where $\lambda_{i}$ is the coefficient of hydraulic resistance of the gas pipeline; $T_{c p}$ is the average temperature of gas in the section; $R e$ is the Reynolds number; $k_{e}$ is the equivalent stiffness of pipes; $\eta$ is the coefficient of dynamic viscosity; $\Delta$ is the ratio of gas density to air density; $z$ is the gas compressibility coefficient.

For the given gas pipeline, we determine the total capacity of the compressor stations, based on the condition of isothermal gas compression:

$$
N_{\Sigma}=N_{1}+N_{2}=Q_{B 1} P_{B 1} \ln \frac{P_{H 1}}{P_{B 1}}+Q_{B 2} P_{B 2} \ln \frac{P_{H 2}}{P_{B 2}}=Q_{B} P_{B} \ln \frac{P_{H 1}}{P_{B 1}} \frac{P_{H 2}}{P_{B 2}}
$$

where $N_{1}$ and $N_{2}$ refer to the capacities of the neighbouring compressor stations; $N_{\Sigma}$ is the total capacity of the compressor stations.

In Equation (1), the product of all numbers in steady isothermal conditions is expressed as $Q_{B 1} P_{B 1}=Q_{B 2} P_{B 2}=Q_{B} P_{B}=$ const. Expressing pressure $P_{B 2}$ by pressure $P_{H 1}$ from the main equation of gas pipelines, we obtain:

$$
N_{\Sigma}=Q_{B} P_{B} \ln \frac{P_{H 2}}{P_{B 1} \sqrt{1-\frac{\lambda \Delta z T_{c p} L_{1} Q^{2}}{\left(0.326 \cdot 10^{-6}\right)^{2} d^{5} P_{H 1}^{2}}}} .
$$

From Equation (2) it is obvious that with an increase in $P_{H 1}$, the total capacity of compressor stations decreases and reaches the minimum value at $P_{H 1}=P_{m a x}$. Thus, from the viewpoint of minimizing energy costs on transporting gas under conditions of insufficient loading of the gas transmission system, it is necessary to choose operating conditions with the maximum allowable pressure at the outlet of the compressor stations. The appropriateness of transporting gas at high pressures, given the necessity to minimize energy costs on transport, has a physical explanation. High pressures enhance the density of gas (with other conditions being equal), while reducing the linear velocity due to the steady flow of gas, the magnitude of which influences the loss of hydraulic 
pressure on friction. A mathematical model of the unsteady, isothermal, one-way gas flow in a pipeline can be presented by the following equations:

$$
\begin{gathered}
\frac{\partial p}{\partial x}+\rho \alpha \frac{\partial}{\partial x}\left(\frac{\omega^{2}}{2 \rho^{2}}\right)+\beta \rho g \frac{\partial h}{\partial x}+\frac{\lambda \omega^{2}}{2 \rho D}+\gamma \frac{\partial \omega}{\partial t}=0, \\
\frac{\partial \omega}{x}+\frac{1}{c^{2}} \frac{\partial p}{\partial t}=0,
\end{gathered}
$$

where $p(x, t)$ is the pressure $(p)$ as a function of the linear coordinate $x$ and time $t ; \omega$ is the linear velocity of gas; $\lambda$ is the coefficient of hydraulic resistance; $\rho$ is the gas density; $D$ is the diameter; $h$ is the geodetic mark of the profile; $c=\sqrt{k z R T}$ is the sound velocity in gas; $\alpha$ is the Coriolis coefficient ( $\alpha=2$ for the laminar flow, and $\alpha=1.1$ for the turbulent flow). The first equation takes into account the friction forces, the difference between heights of the pipeline, and the inertial resistance. The second equation describes the quantitative balance of gas. In this case, changes in temperature depending on the pipeline length are taken into account by means of construction of the iterative algorithm. In system (3), coefficients $\beta$ and $\gamma$ are introduced in order to study the influence of the corresponding forces.

\section{Obtained Results}

\subsection{Oscillatory Process of the Pressure Function in Space and Time}

Neglecting the influence of gravitational and coriolis forces, we reduce system (3) to the following equation:

$$
\frac{\partial^{2} P}{\partial x^{2}}=\frac{2 a}{c^{2}} \frac{\partial P}{\partial t}+\frac{1}{c^{2}} \frac{\partial^{2} P}{\partial t^{2}}
$$

where $2 a$ is the linearization coefficient:

$$
2 a=\frac{\lambda \omega}{2 D}
$$

This equation describes the oscillatory process of the pressure function in space and time, and is known as telegraphic equation in mathematical physics. We note that pressure fluctuations in the gas flow may have different frequencies and amplitudes depending on their cause. Given the above, pressure fluctuations are conventionally divided into high-frequency, medium-frequency, and low-frequency ones. High-frequency fluctuations are characterized by frequencies in the range of $0.4-4.0 \mathrm{~Hz}$, and usually result from a jump-like change in the parameter (pressure, flow) in a certain section of the gas pipeline. The amplitude of such fluctuations can reach $1 \mathrm{MPa}$. Fluctuations propagate along the gas pipeline with the speed of sound, while the amplitude and frequency decrease. The medium-frequency range is $0.5-1.0 \mathrm{~Hz}$. Such fluctuations cause smooth changes in the flow parameters over time. They propagate along the pipeline with a significantly lower decrement of damping. Low-frequency fluctuations are caused by daily unevenness of gas consumption and are in the frequency range of $10^{-5}-0.5 \mathrm{~Hz}$. The amplitude of the pressure variation depends on the nature of the disturbance factor and may be unrestricted (for example, under conditions of filling the gas pipeline with gas). In conditions of high frequency fluctuations, the inertial forces and hydraulic resistance forces in the flow of gas play a decisive role in the formation of the process. Forces of the hydraulic resistance of the pipeline are the main source of the medium- and low-frequency fluctuations. As regards the reliable operation of the gas transmission system, a crucial role is played by high-frequency fluctuations of pressure, given the unpredictable nature of the process. Since the frequency and amplitude of pressure fluctuations caused by disturbance of the gas flow parameters are characteristics of the unsteady process, there must be a link between the amplitude-frequency characteristics and the criterion of unsteadiness [12-14].

Thus, there is an optimization problem, which consists in determining the rational pressures of the steady process in the gas pipeline, under which minimum energy costs on transport would be achieved, on the one hand, and safe operation of pipeline systems would be ensured, on the other hand [15]. As noted, the maximum possible pressure in the gas pipeline allows minimizing the hydraulic losses during the transportation of gas, that is, achieving the minimum energy costs. However, pressure 
fluctuations in unsteady processes caused by a jump-like change in parameters (most often gas flow under insufficient loading) may go beyond the limits of permissible loads. Therefore, it is necessary to choose the maximum possible pressure of the steady process in the gas pipelines, at which the superposition of the pressure amplitude in the unsteady process would not force the load of pipe walls beyond the range of permissible values [16].

The above statement of the problem requires the solution of Equation (4) under the following initial and boundary conditions chosen for the following considerations. Prior to the beginning of an unsteady process caused by disturbance of gas flow, the gas pipeline worked in steady process conditions with the lengthwise distribution of pressure according to a parabolic law.

$$
P(x, 0)=\sqrt{P_{H}^{2}-\left(P_{H}^{2}-P_{K}^{2}\right) x / L},
$$

where $P(x, 0)$ is the pressure at distance $x$ from the beginning of the gas pipeline with length $L$; $P_{H}, P_{K}$ is the pressure at the beginning and at the end of the gas pipeline, respectively.

At given pressures $P_{H}, P_{K}$, a certain mass productivity $Q_{0}$ of the gas pipeline is provided, which can be increased or decreased to a certain magnitude at any time under insufficient loading. We assume that starting from the moment $t>0$, the supply of gas to the gas pipeline has not changed, whereas the offtake of gas at the end of the route has changed by $\Delta Q$. Then the boundary conditions for the realization of Equation (2) will take the following form:

$$
Q(0, t)=Q_{0} ; \quad Q(L, t)=Q_{1},
$$

where $Q_{1}=Q_{0}+\Delta Q$. Align correctly

Using the first equation of system (3) and neglecting all types of power consumption except hydraulic resistance, we obtain:

$$
-{\frac{\partial P}{\partial x_{x=0}}}=\frac{2 a}{F^{2}} Q_{0} ;-\frac{\partial P}{\partial x}{ }_{x=L}=\frac{2 a}{F^{2}} Q_{1}
$$

where $F=\frac{\pi D^{2}}{4}$ is the cross-sectional area of the pipeline.

Equation (4) under the initial (5) and boundary (7) conditions is solved by the Fourier method:

$$
\begin{gathered}
P(x, t)=\frac{\lambda \rho w}{2 d F^{2}} x\left(Q_{0}-\frac{Q_{0}-Q_{L}}{2 L} x\right)+ \\
+\frac{2}{L} \sum_{n=1}^{\infty}\left\{\int_{0}^{L} \sqrt{P_{H}^{2}-\left(P_{H}^{2}-P_{K}^{2}\right) x / L \cos \frac{\pi n x}{L} d x-\frac{\lambda w}{\pi n F}\left[Q_{0}\left(1-(-1)^{n}\right]-\right.}\right. \\
\left.\left.-\frac{1}{2 \pi n}\left(Q_{0}-Q_{L}\right)(-1)^{n}\right]\right\} \exp \left(-\frac{\lambda w}{4 d} t\right) \sin \left[\frac{\lambda w}{4 d} t \sqrt{\left(\frac{4 \pi m c d}{\lambda w}\right)^{2}-1}\right] \cos \frac{\pi n x}{L} .
\end{gathered}
$$

\subsection{Modeling of Unsteady Pressure Fluctuations in the Gas Pipeline with Enabled En-Route Offtake of Gas}

The amplitudes of pressure fluctuations were estimated in time and along the route of the gas pipeline, when disturbances in the form of jump-like changes in productivity occurred at the beginning or at the end of the gas pipeline. We considered pressure fluctuations in the initial section $P(0, t)$, where the greatest values of the absolute pressure in superposition with amplitude fluctuations can exceed the permissible load. Pressure fluctuations depend on a jump-like change in the gas flow as a disturbance factor, as well as on the coordinates of disturbance, absolute values of pressure and temperature, physical properties of gas [12-14]. They were calculated from Equation (8) with different values of the above parameters as model parameters.

It is known that when temperature and other basic physical properties of gas change in the ranges that correspond to the real operating conditions of gas pipelines, their influence on the amplitude of pressure fluctuations is insignificant. The main parameters that determine the amplitude and frequency of pressure fluctuations in the unsteady process caused by a jump-like change in the gas 
flow are the working pressure, the value of the gas flow, and the linear coordinate of the gas offtake. Unsteady processes at different values of working pressure and linear coordinate of the gas offtake are simulated in Figure 1.

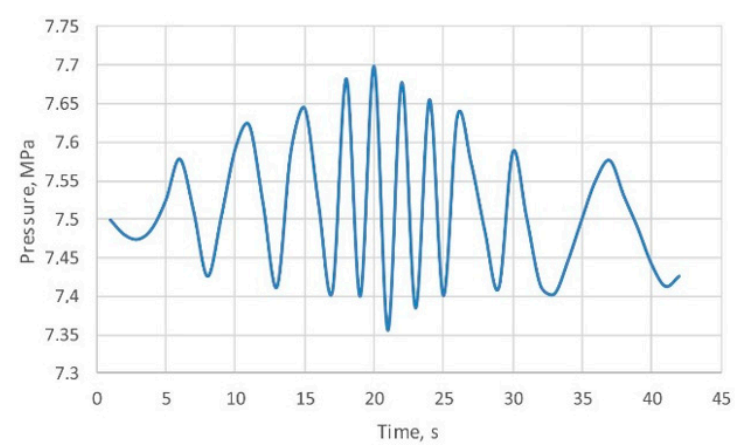

(a)

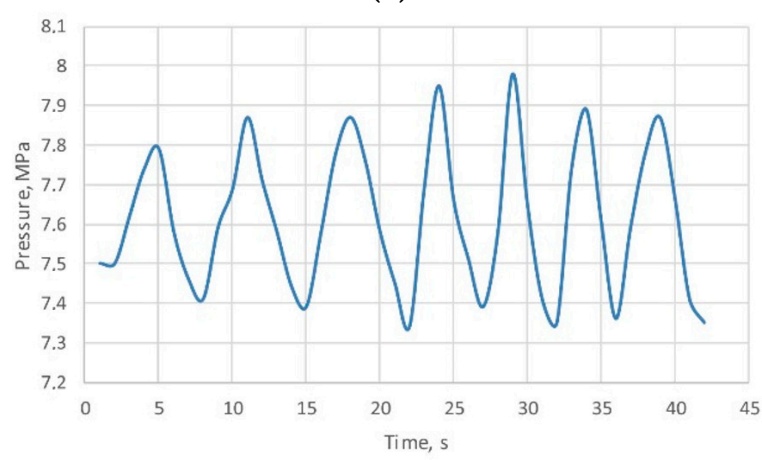

(c)

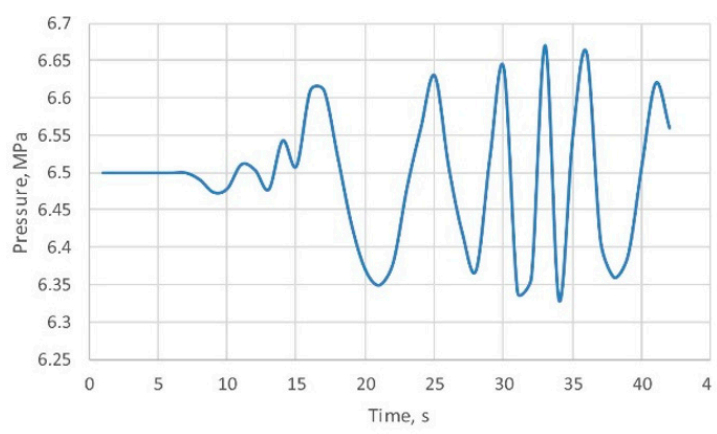

(b)

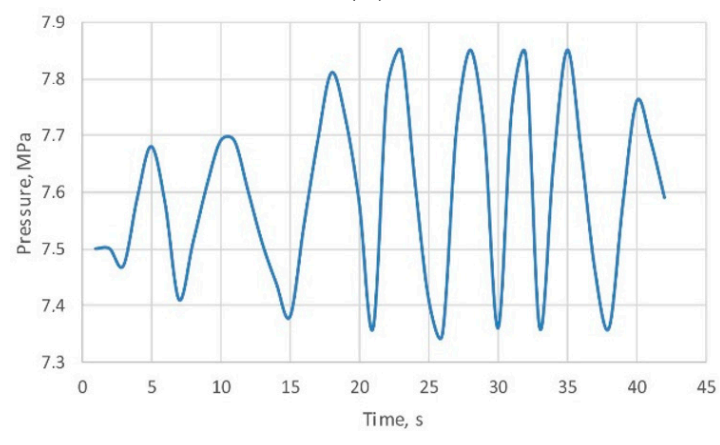

(d)

Figure 1. Results of unsteady process simulation with enabled en-route offtake of gas (pressure fluctuations at the beginning of the linear section): (a) at the initial pressure of $7.5 \mathrm{MPa}$; (b) at the initial pressure of $6.5 \mathrm{MPa}$; (c) at the initial pressure of $7.5 \mathrm{MPa}$, with offtake at the beginning of the section; (d) at the initial pressure of $7.5 \mathrm{MPa}$, with offtake in the middle of the section.

Let us denote the physical properties of gas by the gas constant $R$, and the thermal regime by the absolute temperature $T$. In this case, given the results of the model application, it can be stated that the product $R T$ is a characteristic parameter of the dynamics of the unsteady process, which determines (although not to a large extent) the amplitude and frequency of pressure fluctuations. In other words, when changing the gas constant and the temperature of gas in such a way that their product remains unchanged, the nature of pressure fluctuations in the unsteady process will be the same. On the other hand, the $R T$ product characterizes the propagation rate of small disturbances of gas, that is, the speed of sound $c=\sqrt{k R T}$. Hence, in order to characterize the unsteady process, it is necessary to choose the propagation rate of sound in the gas flow as the independent parameter.

The analysis of results presented in Figure 1 shows that during a jump-like change in the gas flow, the pressure wave propagates with the speed of sound to the initial cross section of the gas pipeline where the pressure is maximal, and causes the oscillatory process, the amplitude and frequency of which increase gradually and reach the maximum after (4-6) periods of oscillation. After that the amplitude and frequency of the process begin to decrease. The total duration of the oscillation process with high values of amplitude and frequency is 50-60 seconds, which is considered as a short-term overload of the pipeline. Subsequently, the amplitude and frequency of pressure fluctuations decrease significantly, and within $0.5-0.583$ hours the oscillation process fades away completely, transferring the operation of the gas pipeline to a new steady regime.

A number of calculations made in accordance with the proposed mathematical model allowed establishing a number of regularities in the oscillation process caused by a jump-like change in the 
gas flow [16]. In particular, it was established that with a decrease in the working pressure in the gas pipeline, the total duration of the high-frequency oscillation process and the unsteady process in general increases, and the frequency and amplitude of oscillations decrease. Thus, when the working pressure is reduced from $7.5 \mathrm{MPa}$ to $7.0 \mathrm{MPa}(6.7 \%)$, the maximum amplitude of pressure fluctuations decreases from $0.199 \mathrm{MPa}$ to $0.18 \mathrm{MPa}$, that is, by $9.5 \%$, and when the working pressure is reduced to $6.5 \mathrm{MPa}$ (by $13.3 \%$ ), the amplitude of pressure fluctuations is $34.7 \%$. The maximum frequency of the oscillation process at a working pressure of $7.5 \mathrm{MPa}$ is $0.44 \mathrm{~Hz}$, and with a decrease in pressure to $7.0 \mathrm{MPa}$ it decreases by $21.8 \%$, and with a further decrease in pressure to $6.5 \mathrm{MPa}$ it decreases by $39.4 \%$. From the physical point of view, this is due to a decrease in the elasticity of the medium, in which oscillating waves propagate, leading to a decrease in the propagation velocity of disturbances and, consequently, to an increase in the duration of the unsteady process and its high-frequency band $[16,17]$.

The linear coordinate of the localized gas offtake has a significant impact on the nature of the unsteady process, in particular, on the amplitude and frequency of pressure fluctuations. When the localized offtake is close to the initial cross-section of the linear region, where the constant pressure is maximal, the amplitude of pressure fluctuations increases, and the frequency decreases. When the localized offtake is in the middle of the linear section of the gas pipeline, the maximum amplitude of the pressure fluctuation is $18.3 \%$ lower than that of the localized offtake located in the initial section of the gas pipeline. If the localized offtake is transferred to the final part of the linear section, the amplitude decreases by $48.5 \%$ under other identical conditions, and the frequency of oscillations varies in a smaller range. So, in the first case (when the localized offtake is in the middle of the linear section), the frequency increases by $7.2 \%$, while in the second case (when the localized offtake is at the end of the linear section) - by $11.4 \%$.

A change in the speed of sound has a lesser influence on the nature of pressure fluctuations in the unsteady process caused by a jump-like change in the gas flow. In addition, an increase in the speed of sound leads to an increase in the amplitude and frequency of oscillations. With an increase in the speed of sound from $400 \mathrm{~m} / \mathrm{s}$ to $440 \mathrm{~m} / \mathrm{s}(10 \%)$, the amplitude increases by $5.7 \%$, and the frequency is $3.1 \%$. With an increase in the speed of sound to $480 \mathrm{~m} / \mathrm{s}$ (by $20 \%$ ), the amplitude increases by $8.5 \%$, and frequencies - by $4.9 \%$.

The value of the localized gas offtake has the greatest influence on the amplitude and frequency of pressure fluctuations at the beginning of the linear section of the gas pipeline in the unsteady process caused by a jump-like change in gas flow. If a jump-like gas flow caused by the localized offtake is $10 \%$ of the total gas flow in the gas pipeline under steady conditions, the maximum amplitude of pressure fluctuations in the unsteady process caused by a sudden leakage will be $0.154 \mathrm{MPa}$. With an increase in the gas flow caused by the localized offtake to $20 \%$, the amplitude of pressure fluctuations increases to $0.287 \mathrm{MPa}$, that is, by 2.45 times. If the gas flow due to the localized offtake is $30 \%$ of the total gas flow in the gas pipeline under steady conditions, the amplitude of pressure fluctuations will be $0.517 \mathrm{MPa}$, that is, it will increase by 3.55 times, while at a jump-like increase in the gas flow due to the localized offtake to $50 \%$ of the gas flow in the gas pipeline under steady conditions, the amplitude of pressure fluctuations will be $1.14 \mathrm{MPa}$, which may endanger the safe operation of the gas pipeline due to a short-term overload.

The maximum frequency of pressure fluctuations in an unsteady process decreases with an increase in the gas flow caused by the localized offtake. With an increase in the gas flow caused by the localized offtake from $10 \%$ to $20 \%$, the maximum frequency of pressure fluctuations decreases by $5.8 \%$, and with a further increase in the gas flow caused by the localized offtake to $30 \%$, the maximum frequency of pressure fluctuations decreases by $12.3 \%$.

\subsection{Ability to Regulate Pressure by Disconnecting Individual Compressor Stations}

The analysis of the results of simulation of an unsteady process in a gas pipeline caused by a jump-like change in the gas flow under conditions of insufficient loading shows that with the maximum 
allowable constant pressure at the beginning of the linear section of the gas pipeline, the amplitude of the pressure fluctuation can lead to a short-term overload of the pipe walls, that is, to an unsafe operation of the gas transmission system. Therefore, when controlling operating conditions, it is necessary to make decisions on providing the permissible pressure at the beginning of the linear section (at the outlet of the compressor station) in cases of a jump-like change in the gas flow. One of the options is disconnecting individual compressor stations. As shown in [18-20], depending on the quantity and serial numbers of the working stations, it is possible to achieve the required performance of the gas transmission system. At the same time, given the low efficiency of gas-pumping units with gas turbine drives, disconnecting individual CSs can be the most effective method of controlling performance from the energetic point of view. It is obvious that this method of control can be used for seasonal regulation of performance, and it should be borne in mind that shutdown and re-start of the CS will require additional energy costs.

However, from the technological point of view, shutdown and re-start of the compressor station may lead to an unsteady process, the duration of which should be predicted in order to provide consumers with gas. It is necessary to take into account the influence of unsteady processes in long-distance gas transmission systems with a large number of compressor stations [12-14]. Prediction and analysis of the said conditions in the gas transmission system, as well as the estimation of energy losses, are possible using the mathematical model of the unsteady gas flow in pipes taking into account an increase in pressure at compressor stations and discontinuity of flow:

$$
-\frac{\partial P}{\partial x}+\sum_{i=1}^{m} \Delta P_{K C i} \delta\left(x-x_{i}\right)=\left(\frac{\partial(\rho w)}{\partial \imath}+\frac{\lambda \rho w^{2}}{2 d}\right) ; \frac{\partial P}{\partial t}=-c^{2} \frac{\partial(\rho w)}{\partial x},
$$

where $P(x, t)$ is the pressure in the gas pipeline as a function of the linear coordinate $x$ and time $t$; $\Delta P_{K C i}$ is an increase in pressure at the compressor station with coordinate $x_{i} ; \delta\left(x-x_{i}\right)$ is the Dirac source function, which simulates an increase in pressure at the compressor station, $\rho$ is the density of gas; $w$ is the linear velocity of gas; $d$ is the internal diameter of the gas pipeline; $\lambda$ is the coefficient of hydraulic resistance.

We note that in order to simulate the fading vibration processes in the gas pipeline, the equation of gas flow should include inertial hydraulic losses and frictional losses. The given system of differential Equations (9) is reduced to the equation:

$$
\frac{\partial^{2} P}{\partial x^{2}}=\frac{1}{c^{2}} \frac{\partial^{2} P}{\partial t^{2}}+\frac{2 a}{c^{2}} \frac{\partial P}{\partial t}+\sum_{i=1}^{m} \Delta P_{K C i} \delta^{*}\left(x-x_{i}\right)
$$

where $\delta^{*}\left(x-x_{i}\right)$ is the linear derivative of the Dirac function from the linear coordinate; $c$ is the speed of sound in gas;

We assume that a gas transmission system with the length $L$ has $m$ intermediate compressor stations, which start to work simultaneously at the point of time $t=0$, and that station $k$ is disconnected at the point of time $t_{1}$. In this case, Equation (10) will take the following form:

$$
\frac{\partial^{2} P}{\partial x^{2}}=\frac{1}{c^{2}} \frac{\partial^{2} P}{\partial t^{2}}+\frac{2 a}{c^{2}} \frac{\partial P}{\partial t}+\sum_{i=1}^{m} \Delta P_{K C i} \delta^{*}\left(x-x_{i}\right)+\Delta P_{K C k} \delta^{*}\left(x-x_{k}\right)\left[\sigma(t)-\sigma\left(t-t_{1}\right)\right]
$$

where $\sigma(t)$ is the single Heaviside function. We assume that at the initial moment of time, the gas pipeline was stopped, and constant pressure $P_{o}$ was maintained throughout its length. Then the initial conditions will be:

$$
t=0, \quad P(x, 0)=P_{0}, \quad \frac{\partial P}{\partial x}=0 .
$$

Starting from the moment $t>0$, constant pressure $P(0, t)=P_{H}$ is maintained at the beginning of the gas pipeline, and constant pressure $P(L, t)=P_{K}$ is maintained at its end. 
For the solution of the mathematical model, integral transformations were used, in particular: Fourier sine-transformation and Laplace transformation [10,11]

The application of inverse transformations of Laplace and Fourier after simple transformations allows us to obtain the dependence of the pressure variation on the length and time of the unsteady process in the following form:

$$
\begin{aligned}
& P(x, t)=P_{0}+\left(P_{H}-P_{K}\right) \frac{x}{L}+\sum_{i=1}^{m} \Delta P_{K C i}\left\{\begin{array}{c}
\left(1-\frac{x}{L}\right) \text { npu } x>x_{i} \\
\left(-\frac{x}{L}\right) \text { npu } x<x_{i}
\end{array}\right\}+ \\
& i \neq k \\
& +\Delta P_{K C k}\left[\sigma(t)-\sigma\left(t-t_{1}\right)\right]\left\{\begin{array}{c}
\left(1-\frac{x}{L}\right) n p u \quad x>x_{i} \\
\left(-\frac{x}{L}\right) n p u x<x_{i}
\end{array}\right\}+\sum_{n=1}^{\infty} C_{n} e^{-a t} f(n, t) \sin \left(\frac{\pi n x}{L}\right)+ \\
& +\frac{2}{\pi} \Delta P_{K C k} \sum_{n=1}^{\infty} \frac{1}{n} \cos \left(\frac{\pi n x_{k}}{L}\right) \sin \left(\frac{\pi n x}{L}\right) e^{-a\left(t-t_{1}\right)} f\left(n, t-t_{1}\right) \sigma\left(t-t_{1}\right) .
\end{aligned}
$$

The first four components of the solution of Equation (12) characterize steady operating conditions of the gas transmission system. The fifth component describes the unsteady process caused by the simultaneous connection of all the compressor stations at the moment $t=0$. The last component modulates the unsteady process caused by the disconnection of the $k$-th compressor station from the moment $t_{1}$. If $t_{1}>>0$, that is, the process that occurs within the pipeline is considered after a significant period of time from the moment of connecting all CSs, the initial unsteadiness will not have a significant impact due to a higher order of smallness of the $e^{-a t}$ multiplier, and the solution to the problem of disconnecting the $k$-th compressor station can be represented as:

$$
\begin{gathered}
P(x, t)=P_{0}+\left(P_{H}-P_{K}\right) \frac{x}{L}+\sum_{\substack{i=1 \\
i \neq k}}^{m} \Delta P_{K C i}\left\{\begin{array}{c}
\left(1-\frac{x}{L}\right) n p u x>x_{i} \\
\left(-\frac{x}{L}\right) n p u x<x_{i}
\end{array}\right\}+ \\
+\frac{2}{\pi} \Delta P_{K C k} \sum_{n=1}^{\infty} \frac{1}{n} \cos \left(\frac{\pi n x_{k}}{L}\right) \sin \left(\frac{\pi n x}{L}\right) e^{-a\left(t-t_{1}\right)} f\left(n, t-t_{1}\right) \sigma\left(t-t_{1}\right) .
\end{gathered}
$$

The solution of Equation (13) describes an unsteady process caused by disconnecting the $k$-th compressor station and does not take into account the unsteadiness of the initial process of connecting all the CSs. Therefore, the countdown can start from the moment of disconnecting the $k$-th compressor station. In this case, we obtain:

$$
\begin{gathered}
P(x, t)=P_{0}+\left(P_{H}-P_{K}\right) \frac{x}{L}+\sum_{i=1}^{m} \Delta P_{K C i}\left\{\begin{array}{c}
\left(1-\frac{x}{L}\right) n p u x>x_{i} \\
\left(-\frac{x}{L}\right) n p u x<x_{i}
\end{array}\right\}+ \\
+\frac{2}{\pi} \Delta P_{K C k} \sum_{n=1}^{\infty} \frac{1}{n} \cos \left(\frac{\pi n x_{k}}{L}\right) \sin \left(\frac{\pi n x}{L}\right) e^{-a t}\left(\cos \left(\sqrt{\left(\frac{\pi n c}{L}\right)^{2}-a^{2}}\right) t+\frac{a}{\sqrt{\left(\frac{\pi n c}{L}\right)^{2}-a^{2}}} \sin \left(\sqrt{\left(\frac{\pi n c}{L}\right)^{2}-a^{2}}\right) t\right) .
\end{gathered}
$$

Equation (14) allows predicting the nature of the unsteady process in long-distance gas transmission systems with a large number of compressor stations caused by the shutdown and re-start of one of the stations.

To estimate the duration of the unsteady process, it is necessary to construct the dependence of fluctuations over the period of the mass flow of gas as the most inertial characteristic in the initial or final section of the gas pipeline $[16,19,20]$.

To this end, we use the equation of the gas flow from system (9). Obviously, for the initial $(x=0)$ or finite cross section $(x=L)$, the delta-function of Dirac is $\delta(x-x)=0$, therefore:

$$
-\frac{\partial P}{\partial x}=\frac{\partial(\rho w)}{\partial \imath}+\frac{\lambda \rho w^{2}}{2 d}
$$


To simplify the computational process, we neglect inertial losses in the initial and final cross-sections, that is, we accept that $\frac{\partial(\rho w)}{\partial t}=0$ [3]. This, of course, is associated with a certain error in the calculation of the mass flow of gas, however, in the forecast calculations, it is not important to determine the absolute value of gas consumption, but the dynamics of its change over time. In addition, by using the linearization of the equation of the gas flow, we obtain:

$m(0, t)=-\frac{\pi d^{3}}{\lambda w} \frac{\partial P}{\partial x}\left|x=0, m(L, t)=-\frac{\pi d^{3}}{\lambda w} \frac{\partial P}{\partial x}\right| x=L$

Using Equation (14) after differentiation we obtain:

$$
\begin{gathered}
m(0, t)=-\frac{\pi d^{3}}{\lambda w}\left(\frac{P_{H}-P_{K}}{L}-\frac{\sum_{i=1}^{m} \Delta P_{K C i}}{L}+\frac{2 L}{\pi^{2}} \Delta P_{K C k} \sum_{n=1}^{\infty} \frac{1}{n^{2}} \cos \left(\frac{\pi n x_{k}}{L}\right) e^{-a t}\left(\cos \left(\sqrt{\left(\frac{\pi n c}{L}\right)^{2}-a^{2}}\right) t+\right.\right. \\
\left.\left.\quad+\frac{a}{\sqrt{\left(\frac{\pi n c}{L}\right)^{2}-a^{2}}} \sin \left(\sqrt{\left(\frac{\pi n c}{L}\right)^{2}-a^{2}}\right) t\right)\right), \\
m(L, t)=-\frac{\pi d^{3}}{\lambda w}\left(\frac{P_{H}-P_{K}}{L}-\frac{\sum^{m} \sum_{K C i}}{i \neq k}+\frac{2 L}{\pi^{2}} \Delta P_{K C k} \sum_{n=1}^{\infty} \frac{(-1)^{n}}{n^{2}} \cos \left(\frac{\pi n x_{k}}{L}\right) e^{-a t}\left(\cos \left(\sqrt{\left(\frac{\pi n c}{L}\right)^{2}-a^{2}}\right) t+\right.\right. \\
\left.\left.\quad+\frac{a}{\sqrt{\left(\frac{\pi n c}{L}\right)^{2}-a^{2}}} \sin \left(\sqrt{\left(\frac{\pi n c}{L}\right)^{2}-a^{2}}\right) t\right)\right) .
\end{gathered}
$$

The obtained dependencies, Figure 2, allow predicting the nature of the mass flow fluctuation over time at the beginning and end of a large-distance gas transmission system, which employs $m$ intermediate compressor stations, caused by shutdown or re-start of the $k$-th compressor station $(k=1,2, \ldots, m)$.

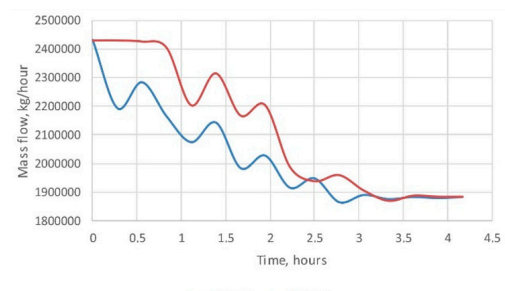

(a)

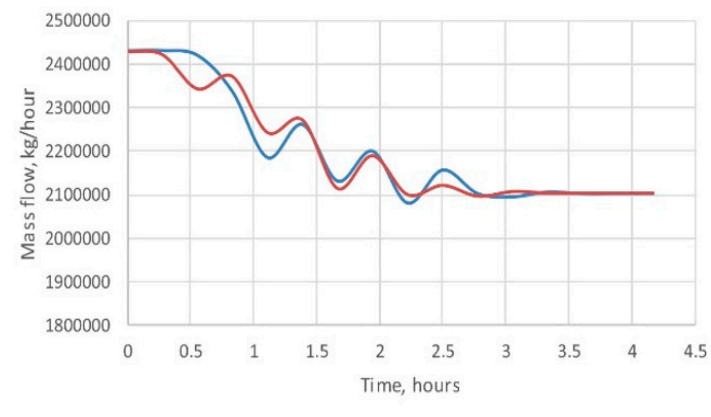

- $\operatorname{cs~9-0~-~} \operatorname{cs} 9-\mathrm{L}$

(c)

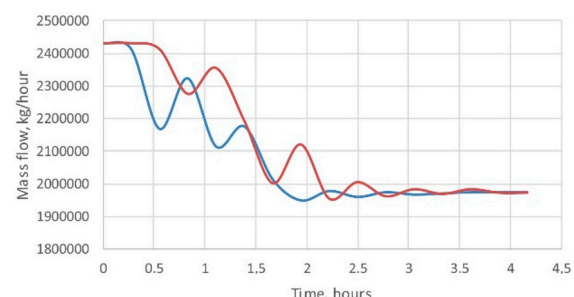

(b)

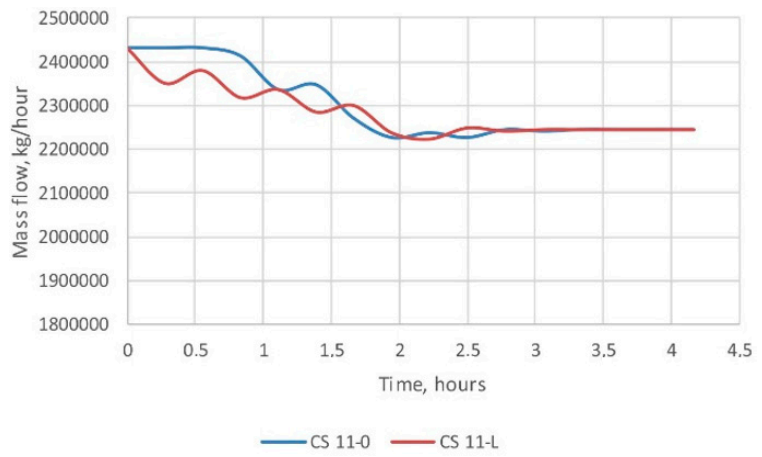

(d)

Figure 2. Regularities in the unsteady process upon shutdown of the CS (fluctuations of mass flow over time): (a) at the beginning of the gas pipeline; $(\mathbf{b}, \mathbf{c})$ at the middle of the gas pipeline; $(\mathbf{d})$ at the end of the gas pipeline.

It is known that oscillatory processes in a gas pipeline create loading conditions different from normal operating condition, which may cause dynamic non-equilibrium processes in the pipe material [21], unpredictable accumulation of local micro-strains, and changes in the crack resistance of 
pipe steels. Therefore, minimizing such negative phenomena is not only a matter of optimizing the gas pumping technology, but also ensuring the integrity of the main gas pipeline.

\section{Discussion of Results}

The gas transportation system (GTS) of Ukraine is the second largest in Europe and one of the largest in the world. The GTS of Ukrtransgas PJSC consists of gas mains (37.6 thousand km long), distribution networks, gas storage facilities, compressor and gas measuring stations (71 compressor stations with a total capacity of $5405 \mathrm{MW}$ ). The throughput at the borders of Ukraine with Russia is 288 billion cubic meters per year, at the borders of Ukraine with Belarus, Poland, Slovakia, Hungary, Romania and Moldova - 178.5 billion cubic meters per year, and with the EU countries - 142.5 billion cubic meters per year [22].

At the same time, most of the gas mains have been in service for more than 30 years, Figure 3a, which requires optimizing their operating conditions and eliminating negative oscillation phenomena, which is particularly important for gas pipelines with a diameter of $1420 \mathrm{~mm}$, the share of which is $16 \%$ of the total amount of gas pipelines, Figure $3 \mathrm{~b}$.

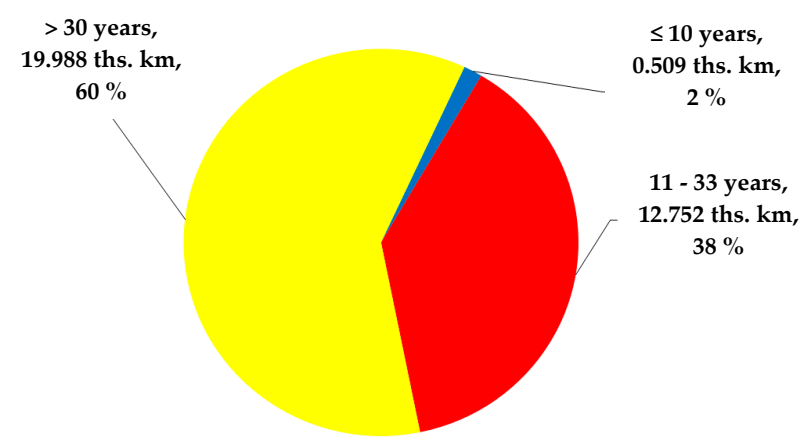

(a)

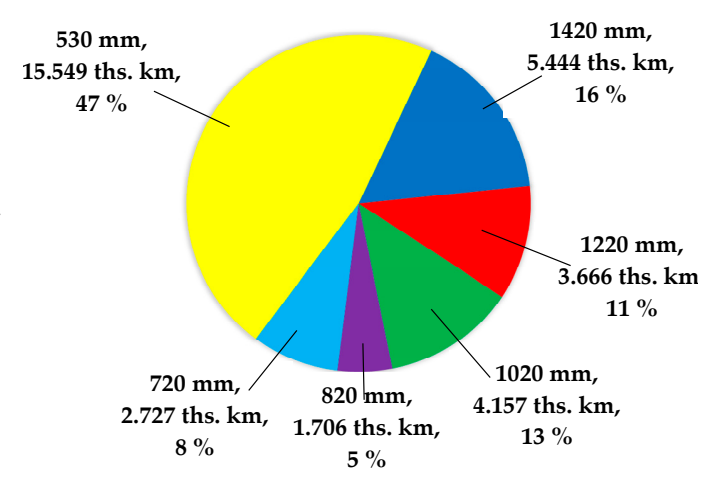

(b)

Figure 3. Structure of the system of gas mains and branches of Ukrtransgas PJSC (ths. km and \%) in terms of the period of operation (a) and diameters (b).

Increasing the reliability of operation of gas mains, which have been in service for more than 30 years, is only possible taking into account the specific features of their laying, and optimization of performance parameters. The main parameters that determine the reliability of gas pipelines are their pressure and temperatures fluctuations.

Insufficient loading of a complex gas transportation system may cause pressure fluctuations in gas pipelines with a given constant productivity. The upper range of possible changes in pressure is limited by the depression line with the maximum initial pressure, and the lower range is limited by the depression line with the minimum final pressure. Violation of the specified range can lead to the pipeline failure due to excess pressure or to failure of centrifugal superchargers of the CS due to lowering of the pressure below the minimum permissible level. The formation of pressure depression at each moment of changing the magnitude of productivity is an unsteady process characterized by pressure fluctuations with a certain frequency and amplitude. The superposition of pressures at the upper limit of depression can lead to an excess of the initial pressure, and at the lower limit of depression - to lowering the pressure below the permissible level.

To study the impact caused by the serial number of the disconnected compressor station on the duration of the unsteady process, a computational experiment was conducted on the basis of the "Soyuz" gas main with a total length of $1567.3 \mathrm{~km}$ (on the territory of Ukraine), a diameter of $1420 \mathrm{~mm}$, and a wall thickness of $20 \mathrm{~mm}$, which has 13 compressor stations equipped with GTK-10I gas-pumping units along its route, Figure 4 [22]. Forecast calculations of the unsteady processes caused by shutdown of the CS were made under the conditions of the project regime, according to which the 
throughput of the gas pipeline is 26 billion $\mathrm{m}^{3}$ per year with the initial pressure (at the outlet of the CS) of 7.5 MPa, and the final pressure (at the inlet of the CS) of 5.0 $\mathrm{MPa}$, which corresponds to the difference $\Delta P_{K C}=2.5 \mathrm{MPa}$ and is the same for all of the CSs.

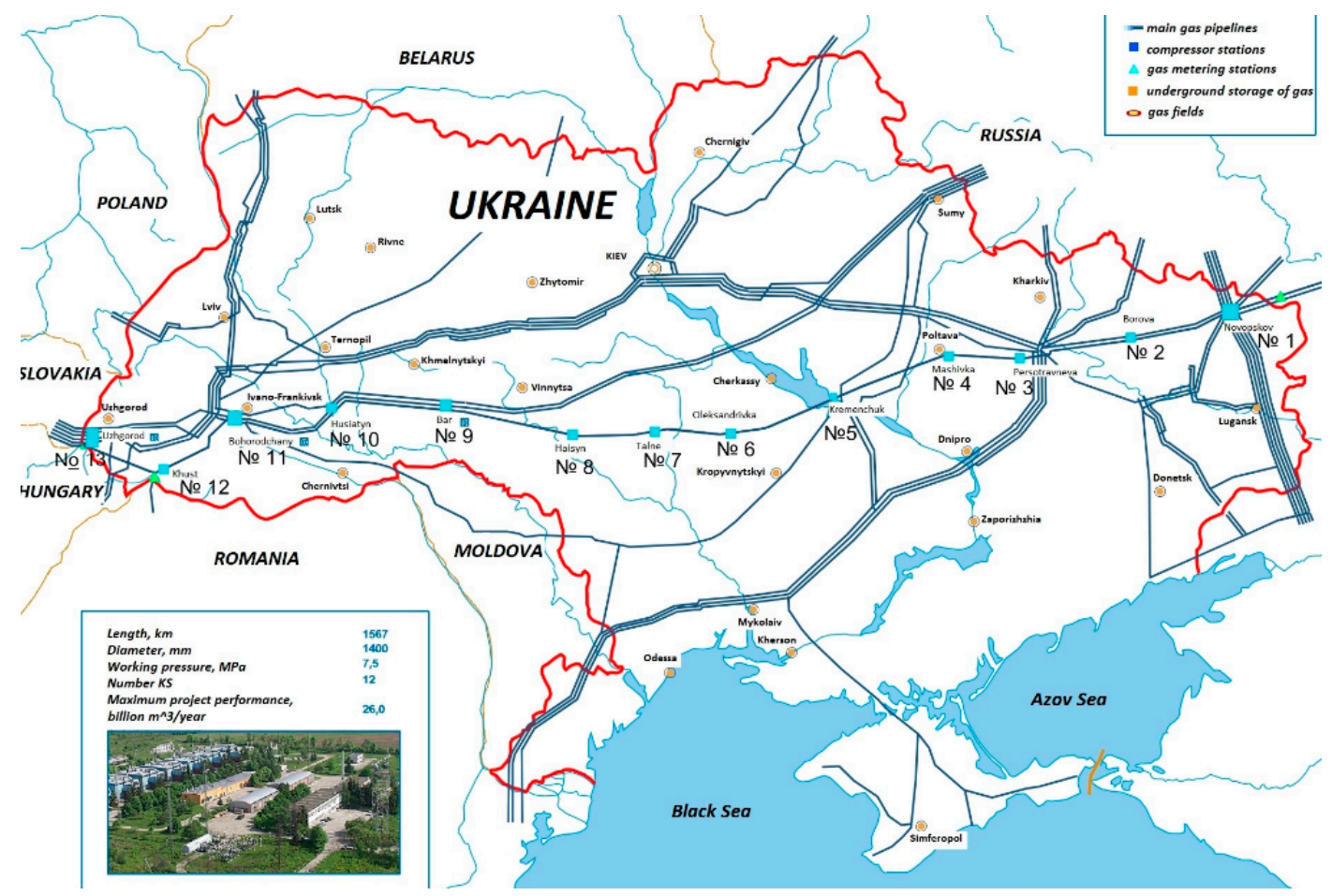

Figure 4. The GTS of Ukraine with the designated "Soyuz" gas main with a total length of $1567.3 \mathrm{~km}$ (the territory of Ukraine), a diameter of $1420 \mathrm{~mm}$, and a wall thickness of $20 \mathrm{~mm}$, which has 13 compressor stations (CS) equipped with GTK-10I gas-pumping units along its route: 1. Novopskov; 2. Borova; 3. Pershotravneva; 4. Mashivka; 5. Kremenchuk; 6. Oleksandrivka; 7. Talne; 8. Haisyn; 9. Bar; 10. Husiatyn; 11. Bogorodchany; 12. Khust; 13. Uzhgorod.

The initial station CS-11 Novopskov was considered to be the main one, and the remaining 12 stations were intermediate. The task was to determine the nature of changes in the gas pipeline performance over time at the beginning and end of the gas pipeline using Equation (15) with the phased disconnection of each intermediate CS.

The analysis of the graphical dependences of the mass gas flow fluctuations at the beginning $(x=0)$ and at the end $(x=L)$ of the gas transportation system has allowed to determine the duration of the unsteady process caused by the phased shutdown of each compressor station. Note that the unsteady processes in gas pipelines are unprofitable in terms of energy consumption for pipelines, as they cause the appearance of inertial forces in the flow of a continuous medium, the work of which leads to a decrease in the overall efficiency of the system. Therefore, the most favorable regime (under other identical conditions) is the one, under which the duration of the unsteady process is minimal.

Calculations using mathematical model (15) show that the longest duration of the unsteady process is possible when the CS-2 Borova is turned off, and is $3 \mathrm{~h} 24 \mathrm{~min} 12 \mathrm{~s}$ at the beginning of the gas transmission system (at the outlet of the Novopskov CS) and 3 hours $42 \mathrm{~min}$ at the end of the system. Thus, the duration of the unsteady process at the end of the system is $30.6 \%$ longer than at the beginning of the system, which is explained by a significant distance between the disconnected CS and the end of the route. When the CS Borova is disconnected, the performance of the new steady process is $22.5 \%$ lower than the throughput (for all working CSs it is $675 \mathrm{~kg} / \mathrm{s}$ ).

When disconnecting the CS Khust (the second one from the end of the route), the duration of the unsteady process is the shortest and is $2.55 \mathrm{~h}$ at the beginning of the gas transmission system 
and 2.26 hours at the end of the route. The duration of the unsteady process at the beginning of the system is $11.3 \%$ longer than at the end of the route, which is explained by the difference in the distance between the disconnected CS and the ends of the pipeline. A decrease in the gas pipeline's performance is $7.6 \%$ compared to the throughput.

Let us consider graphs showing the duration of the unsteady process at the beginning and at the end of the gas transportation system with the phased disconnection of each of the compressor stations, Figure 5. It is noticeable that the duration of the unsteady process caused by the disconnection of a CS decreases at the beginning and end of the gas pipeline with an increase in the serial number of the disconnected station. Moreover, at the beginning of the gas pipeline, a tendency towards decreasing the productivity is less pronounced than at its end. So, when disabling CS-2, the ratio of the duration of the unsteady process at the end of the gas pipeline to the corresponding duration at the beginning of the gas pipeline is 1.306, when disabling CS-5, this ratio is equal to 1.022, and when disabling CS-10, it is 0.935 . This circumstance should be taken into account when planning control procedures for the gas transmission system by disconnecting certain CSs in order to ensure the uninterrupted supply of gas to consumers.

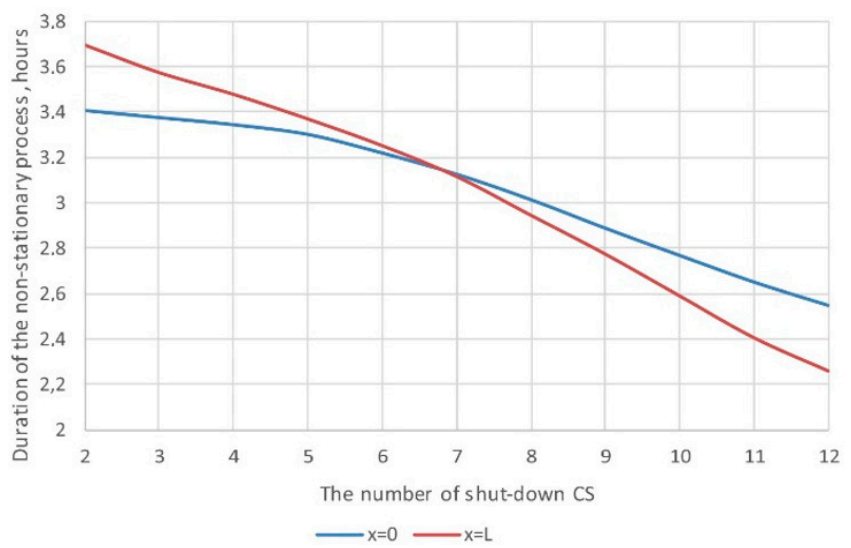

Figure 5. Duration of the unsteady process caused by disconnection of compressor stations for the initial $(x=0)$ and final $(x=L)$ cross-section of the gas pipeline.

At present, the underutilization of the gas transportation system of Ukraine is $42.4 \%$ and it continues to grow [22,23]. Under such conditions, it is almost impossible to prevent unsteady vibrational changes in pressure within the gas pipeline. Therefore, it is necessary to study the amplitude-frequency characteristics of unsteady operating conditions and their duration in order to ensure reliable and efficient operation of gas mains. In $[10,11]$ was found that the pressure difference in the cross-section of the gas pipeline under the obverse and reverse conditions reaches $\sim 20 \%$. This result should be taken into account when scheduling maintenance and in-pipe diagnostics. Planning should also be made taking into account the results of calculating more rigid operating conditions [24].

As of today, the Ukrainian GTS, and, in particular, the "Soyuz" gas main considered, is used for the transit of gas from Russia to the EU [25]. In the event the transit of the Russian gas through the territory of Ukraine is discontinued, this gas pipeline can be transferred to the reverse mode to supply gas to consumers in the east of the country. In this case, the gas pipeline will operate in conditions of insufficient loading, as discussed in this article.

\section{Conclusions}

The mathematical model is developed, which allows estimating the amplitude of pressure fluctuations in the gas pipeline along its length and in time in the presence of disturbances in the form of jump-like changes in productivity at the beginning or at the end of the gas pipeline.

It is proved that the location of the disconnected CS on the route of the gas pipeline has a significant effect on the duration of the unsteady transitional conditions. Moreover, with an increase in 
its serial number within the system, the duration of the unsteady process and the value of a decrease in productivity are reduced.

It is established that with the maximum allowable constant pressure at the beginning of the linear section of the gas pipeline, the amplitude of the pressure fluctuation can lead to a short-term overload of the pipe walls, that is, to an unsafe operation of the gas transmission system. One of the regulation options is disconnecting individual compressor stations.

The dependencies obtained in this research allow predicting the variations of mass flow in time at the beginning and at the end of a long-distance gas transmission system. The method of estimating the parameters of an unsteady process in long-distance gas transmission systems with a large number of compressor stations caused by the shutdown and re-start of one of the stations is proposed.

Author Contributions: Conceptualization, V.Z.; Formal analysis, J.B. and A.G.; Investigation, V.Z., L.P., P.M., V.G.J., R.S., J.B., A.G.; Methodology, V.G.J. and L.P.; Project administration, P.M.; Validation, P.M., R.S., V.Z., and J.B.; Writing—original draft, V.Z., P.M., J.B., L.P., and A.G.; Writing-review and editing, J.B.

Funding: This research was funded by Ministry of Education of the Slovak Republic VEGA No. 1/0424/17 and of the Slovak Research and Development Agency APVV-16-0359 and the APC was funded by Slovak Research and Development Agency.

Acknowledgments: This work was supported by scientific grant agency of the Ministry of Education of the Slovak Republic VEGA No. 1/0424/17 and of the Slovak Research and Development Agency APVV-16-0359.

Conflicts of Interest: The authors declare no conflict of interest.

\section{References}

1. Maruschak, P.; Danyliuk, I.; Prentkovskis, O.; Bishchak, R.; Pylypenko, A.; Sorochak, A. Degradation of the main gas pipeline material and mechanisms of its fracture. J. Civil Eng. Manag. 2014, 20, 864-872. [CrossRef]

2. Maruschak, P.; Poberezhny, L.; Pyrig, T. Fatigue and brittle fracture of carbon steel of gas and oil pipelines. Transport 2013, 28, 270-275. [CrossRef]

3. Doroshenko, Y.; Doroshenko, J.; Zapukhliak, V.; Poberezhny, L.; Maruschak, P. Modeling computational fluid dynamics of multiphase flows in elbow and T-junction of the main gas pipeline. Transport 2019, 34, 19-29. [CrossRef]

4. Vianello, C.; Maschio, G. Quantitative risk assessment of the Italian gas distribution network. J. Loss Prev. Process Ind. 2014, 32, 5-17. [CrossRef]

5. Al-Khalil, M.; Assaf, S.; Al-Anazi, F. Risk-based maintenance planning of cross country pipelines. J. Perform. Construct. Facil. 2005, 19, 124-131. [CrossRef]

6. Shcherbakov, S.G. Problems of Pipeline Transportation of Oil and Gas; Nauka: Moscow, Russia, 1982; 206p. (In Russian)

7. Grudz, V.Y.; Grudz, V.Y., Jr. Forecasting of non-stationary processes in gas transmission systems on condition of their incomplete loading. Naftogazova Energetyka 2017, 28, 62-68. (In Ukrainian)

8. Yakovlev, E.I.; Kazak, O.S.; Myhalkiv, V.B.; Timkov, D.F.; Grudz, V.Y. Modes of Gas Transmission Systems; Lviv: Svit, Ukraine, 1992; 170p. (In Ukrainian)

9. Filipchuk, O.; Grudz, V.; Marushchenko, V.; Myndiuk, V.; Savchuk, M. Development of cleaning methods complex of industrial gas pipelines based on the analysis of their hydraulic efficiency. Eastern-Eur. J. Enterpr. Technol. 2018, 2, 62-71. [CrossRef]

10. Grudz, Y. Formalization of the design model of gas-main pipelines infrastructure failure. Metall. Mining Ind. 2015, 292, 79-84.

11. Grudz, V.Y.; Grudz, V.Y., Jr.; Zapukhlyak, V.B.; Kyzymyshyn, Y.V. Non-stationary processes in the gas transmission systems at compressor stations shut-down. J. Hydrocarbon Power Eng. 2018, 5, 22-27.

12. Meshalkin, V.P.; Chionov, A.M.; Kazak, A.S.; Aristov, V.M. A computer model of the nonstationary gas flow in a long multilayer-insulated high-pressure subsea gas pipeline. Doklady Chem. 2016, 469, 241-244. [CrossRef]

13. Meshalkin, V.P.; Chionov, A.M.; Kazak, A.S.; Aristov, V.M. Applied computer model of the non-stationary gas flow in a long multilayer-insulated high-pressure subsea gas pipeline. Doklady Chem. 2016, 470, $279-282$. [CrossRef] 
14. Meshalkin, V.P.; Moshev, E.R. Modes of functioning of the automated system "pipeline" with integrated logistical support of pipelines and vessels of industrial enterprises. J. Mach. Manuf. Reliab. 2015, 44, 580-592. [CrossRef]

15. Rios-Mercado, R.Z.; Borraz-Sanchez, C. Optimization problems in natural gas transportation systems: A state-of-the-art review. Appl. Energy 2015, 147, 536-555. [CrossRef]

16. Prytula, N.M.; Gryniv, O.D.; Dmytruk, V.A. Simulation of nonstationary regimes of gas transmission system operation. Math. Model. Comput. 2014, 1, 224-233.

17. Szoplik, J. The steady-state simulations for gas flow in a pipeline network. Chem. Eng. Trans. 2010, 21, 1459-1464. [CrossRef]

18. Herrán-González, A.; De La Cruz, J.M.; De Andrés-Toro, B.; Risco-Martín, J.L. Modeling and simulation of a gas distribution pipeline network. Appl. Math. Model. 2009, 33, 1584-1600. [CrossRef]

19. Woldeyohannes, A.D.; Majid, M.A.A. Simulation model for natural gas transmission pipeline network system. Simulat. Model. Pract. Theory 2011, 19, 196-212. [CrossRef]

20. Chaczykowski, M.; Zarodkiewicz, P. Simulation of natural gas quality distribution for pipeline systems. Energy 2017, 134, 681-698. [CrossRef]

21. Chausov, M.G.; Maruschak, P.O.; Pylypenko, A.P.; Prentkovskis, O. Effect of impact-oscillatory loading on variation of mechanical properties and fracture toughness of pipe steel 17G1S-U. In Proceedings of the 20th International Conference 'Transport Means 2016', Vilnius, Lithuania, 5-7 October 2016; pp. 441-443.

22. Maruschak, P.; Prentkovskis, O.; Bishchak, R. Defectiveness of external and internal surfaces of the main oil and gas pipelines after long-term operation. J. Civil Eng. Manag. 2016, 22, 279-286. [CrossRef]

23. Eser, P.; Chokani, N.; Abhari, R. Impact of Nord Stream 2 and LNG on gas trade and security of supply in the European gas network of 2030. Appl. Energy 2019, 238, 816-830. [CrossRef]

24. Maruschak, P.O.; Bishchak, R.T.; Danyliuk, I.M. Crack Resistance of Materials and Structures: Gas Mains after Long-Term Operation; ZAZAPRINT: Ternopil, Ukraine, 2016; 182p.

25. Richter, P.M.; Holz, F. All quiet on the eastern front? Disruption scenarios of Russian natural gas supply to Europe. Energy Policy 2015, 80, 177-189. [CrossRef]

(C) 2019 by the authors. Licensee MDPI, Basel, Switzerland. This article is an open access article distributed under the terms and conditions of the Creative Commons Attribution (CC BY) license (http://creativecommons.org/licenses/by/4.0/). 